\title{
Prevalence estimations of comorbid attention deficit hyperactivity disorder and developmental co-ordination disorder in children aged 8 - 9 in KwaZulu-Natal, South Africa
}

\author{
P M T Dawson, ${ }^{1} \mathrm{PhD}$ (Physio); T Nadasan, ${ }^{2} \mathrm{PhD}$ (Public Admin); P Govender, ${ }^{3} \mathrm{PhD}(\mathrm{OT})$ \\ ${ }^{1}$ Private practitioner, Durban, South Africa \\ ${ }^{2}$ Discipline of Physiotherapy, School of Health Sciences, University of KwaZulu-Natal, Durban, South Africa \\ ${ }^{3}$ Discipline of Occupational Therapy, School of Health Sciences, University of KwaZulu-Natal, Durban, South Africa
}

Corresponding author: P Govender (naidoopg@ukzn.ac.za)

\begin{abstract}
Background. Children diagnosed with attention deficit hyperactivity disorder (ADHD) often encounter motor co-ordination difficulties. Little has been documented about the prevalence of comorbid ADHD and developmental co-ordination disorder (DCD), both in South Africa (SA) and globally.

Objective. The purpose of the study was to establish the prevalence and demographics of comorbid ADHD and DCD at 5 sites located within 3 districts of KwaZulu-Natal Province, SA.

Methods. A descriptive observational study was undertaken of 151 children aged 8 - 9 years, both male and female, from 4 population groups. The children were clinically diagnosed with ADHD and were receiving remedial education. Children with any other conditions and/or receiving occupational therapy, physiotherapy and/or psychotherapy were excluded. The Conners' Teacher Rating Scale, the DCD Questionnaire and the Movement Assessment Battery for Children were administered to confirm comorbid ADHD and DCD.

Results. A 74\% prevalence of comorbid ADHD and DCD was identified, with a male-to-female ratio of 2:1. The combined subtype of ADHD was most commonly associated with comorbid DCD.

Conclusion. From the relatively high prevalence figures, it appears that an association between ADHD and DCD exists. Children with ADHD should therefore be assessed for motor skills deficiency and offered appropriate interventions.
\end{abstract}

S Afr J Child Health 2020;14(4):180-184. https://doi.org/10.7196/SAJCH.2020.v14i4.1676

Attention deficit hyperactivity disorder (ADHD) is a prevalent neurodevelopmental disorder with worldwide prevalence figures of $7-10 \%{ }^{[1,2]} \mathrm{ADHD}$ is characterised by 3 core symptoms, namely inattention, impulsivity and hyperactivity, which result in impairments in social, academic and occupational settings. ${ }^{[1,2]}$ Developmental co-ordination disorder (DCD) is a neurodevelopmental disorder characterised by delayed motor skills development. The onset is early and affects academic achievement, activities of daily living (ADL), sport and leisure. International prevalence figures of DCD range from $5-15 \% \cdot{ }^{[1,3-5]}$ World prevalence rates of DCD range from 5 - $8 \%$ within the 6 - 12 -year age group. ${ }^{[1]}$

In addition to the 3 core symptoms of $\mathrm{ADHD}$, impairments in co-ordination, balance, motor planning and executive functions have been identified in children diagnosed with ADHD. ${ }^{[6-10]}$ Symptoms of DCD include reduced muscle tone and muscle power, impaired gross and fine motor co-ordination, balance deficits, visual-motor problems, poor core stability, bilateral and sensory integration problems, postural anomalies and motor planning deficits. ${ }^{[3-5]}$ The condition of ADHD is associated with a host of comorbid conditions, the most commonly occurring deficits being motor developmental problems, which occur in $60 \%$ of cases. ${ }^{[6-9]}$ A number of conditions co-exist with DCD, the most common being ADHD, which occurs in $50-60 \%$ of cases. ${ }^{[3,11-14]}$ Based on the available literature, comorbid ADHD and DCD exist commonly. ${ }^{[15]}$ However, prevalence in the context of South Africa (SA) has not been determined. In the present study, the authors attempted to establish prevalence figures and demographics of children aged 8 - 9 years presenting with comorbid ADHD and DCD.

\section{Literature review}

Children with ADHD often experience motor co-ordination deficits. ${ }^{[1]}$ Fliers et al.$^{[16]}$ conducted a study in the Netherlands on 486 children, aged $5-18$ years, diagnosed with ADHD. Parents completed a DCD questionnaire (DCDQ), after which the Groningen Motor Observation Scale was administered to all children. Results revealed a 33\% prevalence of comorbid ADHD and DCD. This prevalence was similar in both genders, and motor problems were most commonly identified in the inattentive type of ADHD. ${ }^{[16]}$ Watemberg et al. ${ }^{[15]}$ completed a study in Israel, where the Movement Assessment Battery for Children (MABC) was administered to 96 children with ADHD who were between 6 and 12 years old. Test results revealed that 53 children had DCD, and a $55.2 \%$ prevalence of comorbid ADHD and DCD was found. As in Fliers' study, ${ }^{[16]}$ comorbid ADHD and DCD was most prevalent in the inattentive type of ADHD, but followed closely by the combined type ${ }^{[15]}$ Three SA studies ${ }^{[8,17,18]}$ were found, the first of which tested 528 children, aged 6 - 9 years, diagnosed with ADHD, in 7 ethnic groups on 3 fine-motor tests. Prevalence figures were not explicitly recorded. There was no difference found between boys and girls, and the combined subtype of ADHD was most 
affected. ${ }^{[8]}$ The second study tested efficacy of treatment methods for ADHD with associated motor difficulties, but failed to establish prevalence figures and present demographics of children with comorbid ADHD and DCD. ${ }^{[17]}$ The third and most recent SA study tested several motor skills in 160 children ( 80 with ADHD and 80 controls) aged $7-13$ years. Children with ADHD displayed poorer scores in all motor tasks than did the control group. ${ }^{[18]}$ Once more, demographics describing comorbid ADHD and DCD were not outlined. The paucity of information on prevalence figures and demographics of comorbid ADHD and DCD worldwide, and in SA in particular, is evident. There is neuroanatomical evidence to suggest that comorbid ADHD and DCD occur, in that certain areas of the brain control both motor and executive/cognitive functions, namely the frontal region, cerebellum, basal ganglia and thalamus. ${ }^{[3,6,19,20]}$ Levels of neurotransmitter substances such as dopamine, norepinephrine and serotonin are also considered to be reduced in both ADHD and DCD. ${ }^{[3,6,19,20]}$

In $\mathrm{SA}, \mathrm{ADHD}$ is presently diagnosed by general practitioners (GPs), paediatricians and educational psychologists. Children are often managed with neuropharmacological drugs. Often, these children are not screened for motor or co-ordination deficits and hence the intervention for these deficits is not provided. Clearly, the association between the two conditions needs to be explored, to provide a more holistic approach to management of children presenting with comorbid ADHD and DCD.

\section{Methods \\ Study method}

This was a descriptive observational study undertaken to determine the prevalence of comorbid ADHD and DCD in children aged 8 - 9 years. Demographics such as average age, gender, population group figures and subtype of ADHD were recorded.

\section{Population, recruitment and sampling procedures}

The children were primary school children, aged $8-9$ years and 11 months, from any population group, who were receiving remedial education in mainstream schools. All mainstream schools with remedial education units in KwaZulu-Natal (KZN) were invited to participate in the study $(N=23)$. The school became a study site once the principal had signed consent. The final sample consisted of children from 4 mainstream schools, as well as children assessed at a private practice that had children fed in from mainstream schools with remedial education units. Children included in the study were recently clinically diagnosed with ADHD, within a maximum of 3 months of being recruited into the study. Children having started neuropharmacological medication were included, as it would be unethical to discontinue this treatment. Children having any other neurological, psychiatric, orthopaedic, intellectual or genetic disorders were excluded from the study. Any child who had started any form of therapy (occupational therapy, physiotherapy and/or psychotherapy) was also excluded from the study. Parental consent and child assent was received for 151 children from the 5 sites for participation in the study.

\section{Data collection tools}

The Modified Conners' Teacher Rating Scale ${ }^{[21]}$ was used to confirm the diagnosis of ADHD. This scale was developed on children aged 6 - 18 years and consists of 20 items. Teachers were requested to rate children who were not on any neuropharmacological medication. Each item is rated on a 4-point Likert scale and scored as follows:
- not at all (0) - 0 points awarded

- just a little (1) - 0.5 points awarded

- pretty much (2) - 1 point awarded

- very much (3) - 1.5 points awarded.

The first 10 items relate to inattention. The next 5 items relate to impulsivity, and the final 5 items relate to hyperactivity. Percentages are calculated for each core symptom, and are interpreted as follows: - $60-70 \%$ mild case of inattention/impulsivity or hyperactivity

- greater than $70 \%$ moderate to severe case of inattention/ impulsivity/hyperactivity.

From these percentages, 3 subtypes are identified: inattentive, hyperactive-impulsive or combined (inattention/impulsivity/ hyperactivity).

The DCDQ is a parent-rated questionnaire, used to identify DCD in children aged 5 - 15 years. It consists of 15 motor/co-ordination items rated on a 5-point Likert scale and provides a measure of co-ordination in daily functional activities. ${ }^{[22]}$ The total was interpreted as follows, for children within the age band of 8 years to 9 years and 11 months:

- 15 - 55 points - DCD

- 56 - 75 points - not DCD.

The MABC version 1 (MABC-1) $)^{[23]}$ was used to derive a quantitative assessment of DCD as the authors felt it to be more appropriate for ages 8 - 9 years, with age bands not as wide as the MABC-2. ${ }^{[23]}$ The test has 8 items, which focus on 3 main areas, namely manual dexterity, eye-hand co-ordination and balance. The test was administered by the first author or a research assistant. Total scores were interpreted as follows:

- motor score $<10.5$ - not a DCD diagnosis

- motor score $>10.5$ - DCD diagnosis.

DCDQ and MABC-1 have concurrent validity and therefore the ability to diagnose DCD. ${ }^{[22,23]}$

\section{Procedure}

Teachers and parents of the children recruited for this study were orientated and provided with guidance on how to complete the Conners' Teacher Rating Scale and the DCDQ, respectively. The MABC-1 was administered by the first author, during school hours, in a classroom or therapy room that was conducive to testing of gross motor skills. None of the children was on neuropharmacological drug therapy at the time of testing. On the basis of a child having all 3 tests positive, they were classified as having comorbid ADHD and DCD. Prior to commencement of the main study, a pilot study was conducted on 20 children. The testing procedure followed the same protocol as that for the main study. The pilot study was useful in that it confirmed the clinical diagnosis of ADHD with use of the Conners' Teacher Rating Scale. A DCD diagnosis was confirmed by using scores derived from the MABC- 1 and $\mathrm{DCDQ}$.

\section{Statistical analysis}

Demographic data were entered onto an Excel spreadsheet which was then imported into the SPSS version 25.0 (IBM Corp., USA). Average age was determined by the mean and standard deviation. The statistics were represented in tabular form. The ShapiroWilk $t$-test was used to test the data for normality. Frequency tables and percentages were used to determine the prevalence of comorbid ADHD and DCD, gender distribution, racial population 
group percentages and ADHD subtype (inattentive/hyperactiveimpulsive/combined). Cross-tabulations of gender and population group with subtype of ADHD were also completed.

\section{Ethical considerations}

The Biomedical Research Ethics Committee of the University of KZN approved the study (ref. no. BFC397/16). The KZN Department of Education also approved the study. Consent was obtained from the school principals and parents, and assent from the children.

\section{Results}

A total of 151 children were tested. Of these, 112 (74\%) tested positive and were diagnosed with comorbid ADHD and DCD (Table 1). Of these 112,74 boys (66\%) and 38 girls (34\%) were diagnosed with comorbid ADHD and DCD, with a male-to-female ratio of 2:1. Of the children presenting with comorbid ADHD and DCD, $4.5 \%$ were SA Asian children, $49 \%$ were SA black children, $6.3 \%$ were SA coloured children, and $40.2 \%$ were SA white children (Table 1).

Of the 112 children diagnosed with comorbid ADHD and DCD, $42 \%$ children presented with the inattentive type of ADHD, $1.8 \%$ had the hyperactive/impulsive type, and $56.2 \%$ had the combined type. The mean average age of the children was 8.8 years (range 7.8 - 9.9 years; standard deviation 0.6). Cross-tabulation revealed a greater percentage of boys presenting with the combined subtype of ADHD (38.4\%) followed by inattentive type (26.8\%), in comparison with girls (Table 2). More SA black children (49.1\%) with ADHD presented than did the other population groups. Of these, $24.1 \%$ presented with the inattentive subtype of ADHD more than the combined type (23.2\%). More SA white children presented with the combined type (25.9\%) than the inattentive type (14.3\%) (Tables 2 and 3).

\section{Discussion}

The purpose of the present paper was to establish the prevalence and present the demographics of children, aged 8 - 9 years, with comorbid ADHD and DCD. As illustrated by the results, 74\% of children presented with comorbid ADHD and DCD. This figure is higher than the figures presented in the current available literature. For example, a prevalence of comorbid ADHD and DCD of $55.2 \%$ was indicated in a study sample in Israel, ${ }^{[15]}$ with a $50 \%$ prevalence in Australia ${ }^{[24]}$ and $33 \%$ in children in a study in

Table 1. Prevalence figures and demographics of comorbid ADHD and DCD

\begin{tabular}{|c|c|c|c|c|}
\hline Measure & Comorbid ADHD and DCD cases (A), $n$ & Total tested (B), $n$ & A/B (\%) & $\mathrm{A} / 112(\%)$ \\
\hline Prevalence of comorbid ADHD and DCD & 112 & 151 & 74 & - \\
\hline \multicolumn{5}{|l|}{ Gender } \\
\hline Male & 74 & 103 & 71 & 66 \\
\hline Female & 38 & 48 & 79 & 34 \\
\hline \multicolumn{5}{|l|}{ Population group } \\
\hline Asian & 5 & 5 & 100 & 4.5 \\
\hline Black & 55 & 74 & 74 & 49 \\
\hline Coloured & 7 & 9 & 78 & 6.3 \\
\hline White & 45 & 63 & 71 & 40.2 \\
\hline \multicolumn{5}{|l|}{ ADHD subtype } \\
\hline Inattentive & 47 & - & - & 42.0 \\
\hline Hyoeractive-impulsive & 2 & - & - & 1.8 \\
\hline Combined & 63 & - & - & 56.2 \\
\hline
\end{tabular}

Table 2. Cross-tabulation of gender with subtype of ADHD and comorbid DCD

\begin{tabular}{llll}
\hline & \multicolumn{3}{c}{ ADHD subtype } \\
\cline { 2 - 4 } Gender & Inattentive, $\boldsymbol{n}(\%)$ & Hyperactive-impulsive, $\boldsymbol{n}(\%)$ & Combined, $\boldsymbol{n}(\%)$ \\
\hline Male & $30(26.8)$ & $1(0.9)$ & $43(38.4)$ \\
Female & $17(15.2)$ & $1(0.9)$ & $20(17.8)$ \\
Total & $47(42.0)$ & $2(1.8)$ & $63(56.2)$ \\
ADHD $=$ attention deficit hyperactivity disorder; DCD = developmental co-ordination disorder.
\end{tabular}

Table 3. Cross-tabulation of population group with subtype of ADHD and comorbid DCD

\begin{tabular}{|c|c|c|c|c|}
\hline \multirow[b]{2}{*}{ Population group } & \multicolumn{3}{|c|}{ ADHD subtype } & \multirow[b]{2}{*}{ Total $(N(\%))$} \\
\hline & Inattentive $(n(\%))$ & Hyperactive-impulsive $(n(\%))$ & Combined $(n(\%))$ & \\
\hline Asian & $3(2.7)$ & $0(0)$ & $2(1.8)$ & $5(4.5)$ \\
\hline Black & $27(24.1)$ & $2(1.8)$ & $26(23.2)$ & $55(49.1)$ \\
\hline Coloured & $1(0.9)$ & $0(0)$ & $6(5.3)$ & $7(6.2)$ \\
\hline White & $16(14.3)$ & $0(0)$ & $29(25.9)$ & $45(40.2)$ \\
\hline Total (\%) & $47(42.0)$ & $2(1.8)$ & $63(56.2)$ & $112(100)$ \\
\hline
\end{tabular}


the Netherlands. ${ }^{[16]}$ Possible reasons for the difference in statistics include variability in the sample size from $96^{[15]}$ to 151 (in the present study) to 486 children. ${ }^{[16]}$ Moreover, these studies, ${ }^{[15,16,24]}$ which are the only similar studies that have been located that provide actual prevalence percentages, were published between 8 and 10 years ago. Furthermore, the higher prevalence established in the present study could be due to the fact that 3 instruments were administered to confirm the existence of comorbid ADHD and DCD, whereas other studies utilised the DCDQ and Groningen Motor Observation Scale, MABC-1 or MABC-2 independently, and testing of fine motor skills and testing of gross motor skills. A combination of $\geq 3$ tests did not appear to have been utilised. Our study revealed a greater number of boys diagnosed with comorbid ADHD and DCD than girls, in a ratio of 2:1. The previous studies that established comorbid ADHD and DCD cited no gender differences in the prevalence figures. ${ }^{[8,16]}$ A number of literary sources support the findings of our study, in which more boys are affected by ADHD, DCD and by implication the two conditions, in comorbidity. ${ }^{[1,3,6,21]}$

Our study established that the highest percentage of children with comorbid ADHD and DCD was $49 \%$ among the black children, and the lowest percentage was among the SA Asian children, at $4.5 \%$. However, when looking at each population group separately, the SA Asian children had the highest percentage, with 5 out of 5 children diagnosed with comorbid ADHD and DCD. This disparity in the statistics can be attributed to the small sample of SA Asian children recruited in the present study; the same disparity exists among SA coloured children in the study.

The present study established that, of the 3 subtypes of ADHD, the combined subtype (inattention, impulsivity and hyperactivity) occurs most commonly with DCD. This finding is not supported by the prior 2 studies, which established that the inattentive subtype of ADHD most commonly displays symptoms of DCD. ${ }^{[15,16]}$ Meyer et al. ${ }^{[8]}$ however, conducted a study in SA and also found the combined subtype of ADHD to co-exist most commonly with DCD. ${ }^{[8]}$

The cross-tabulation that highlighted more boys presenting with both inattentive and combined subtypes of ADHD, with comorbid DCD, is supported by the available literature: that a male-to-female ratio of comorbidity of 2:1 seems to exist. ${ }^{[8]}$ The cross-tabulation that revealed a larger proportion of black children presenting with the inattentive subtype and a larger proportion of white children with the combined subtype, has not been previously indicated in the literature. A paucity of literature on comparative ethnic group figures therefore exists.

A strength of the present study was that the ADHD diagnosis was not only confirmed by a Conners' Teacher Rating Scale, but was also provided as a clinical diagnosis (by a paediatrician, psychologist or psychiatrist). Similar studies in SA did not confirm an ADHD diagnosis that had been made clinically. ${ }^{[8,17,18]}$ A second strength was that DCD was diagnosed with both the DCDQ and MABC1 , which demonstrate concurrent validity. ${ }^{[22,23]}$ No similar studies assessed DCD using both instruments. Watemberg et al. ${ }^{[15]}$ and Bart et al. ${ }^{[25]}$ used the MABC-1 alone, while Pearsall-Jones et al..$^{[24]}$ utilised a less well-known motor test (the McCarron Assessment and Neuromuscular Development (MAND)) and Stray et al. ${ }^{[26]}$ used the Motor Function Neurological Assessment (MFNU). A study in the Netherlands used both the DCDQ and the Groningen Motor Observation Scale; both are qualitative measures. ${ }^{[16]}$ A third strength of our study was that all demographic information on children diagnosed with comorbid ADHD and DCD was presented, namely prevalence, gender distribution, mean age, population figures and subtype of ADHD. Other similar studies either did not illustrate prevalence figures, did not establish population group statistics or did not discuss the most commonly affected subtype of ADHD. ${ }^{[8,16,17,24-26]}$ A limitation of this consideration, however, was that long-term effects of neuropharmacological drugs could not be eliminated and may have confounded the baseline scores.

\section{Conclusion}

The present study provided significant statistics and demographics on comorbid ADHD and DCD in three districts of KwaZuluNatal, South Africa. Such statistics have previously not existed and may assist clinicians in the planning and provision of services for such children. Given these findings, evaluation of motor skills among children with ADHD becomes essential. The association between ADHD and DCD, established in this study, suggests that assessment and intervention should focus on symptoms of inattention/impulsivity/hyperactivity as well as motor co-ordination symptoms. Children with ADHD should therefore be referred to a team who would be able to assess, diagnose and manage both of these conditions in comorbidity.

Declaration. This manuscript emanated from a $\mathrm{PhD}$ study that the first author (PMTD) has completed at the University of KwaZulu-Natal.

Acknowledgements. The authors extend their sincere appreciation to Dr O Olagbegi for statistical support, C Dawson for research assistance, and the parents and children who participated in this study.

Author contributions. The study was initiated by PMTD, and conceptualised and designed in consultation with TN and PG, as supervisors of the study. PMTD was responsible for data collection. All authors assisted in interpreting the findings. PMTD was responsible for drafting the first version of the manuscript. TN and PG provided critique and assisted in revising the paper through the review process. All authors approved the final version.

Funding. PMTD received a partial fee remission for study towards a $\mathrm{PhD}$ (Physiotherapy) degree from the University of KwaZulu-Natal. This paper emanated from the $\mathrm{PhD}$ study.

Conflicts of interest. None.

1. Diagnostic and Statistical Manual of Mental Disorders (DSM-IV). Washington DC:American Psychiatric Association; 1994

2. Kern A, Amod Z, Seabi J, Vorster A. South African foundation phase teachers perceptions of ADHD at private and public schools. Int J Environ Res Pub Health 2015;12(3):3042-3059. https://doi.org/10.3390/ijerph120303042

3. Nelson SL, Pataki C. Developmental Coordination Disorder. Medscape reference website. 2015.

4. Gritzman T. An evaluation of teachers' ability to identify children with motor difficulties. (Master's dissertation, MPhysio, Faculty of Health Sciences, University of the Witwatersrand). 2011. wiredspace.wits.ac.za (accessed 29 October 2019).

5. Missiuna C, Gaines R, Mclean J, DeLaat D, Egan M, Soucie H. Description of children identified by physicians as having developmental coordination disorder. Dev Med Child Neurol 2008;50(11):839-844. https://doi.org/10.1111/j.14698749.2008.03140.x

6. Barkley RA. ADHD: Nature, Course, Outcomes and Comorbidity. Courses for Mental Health Professionals. 2015. https://www.continuingedcourses.net/active/ courses/course082.php

7. Nigg JT. Temperament and developmental psychopathology. J Child Psychol Psychiatry 2006;47(3-4):395-422. https://doi.org/10.1111/j.14697610.2006.01612.x

8. Meyer A, Sagvolden T. Fine motor skills in South African children with symptoms of ADHD: Influence of subtype, gender, age, and hand dominance. Behav Brain Funct 2006;2(1):33. https://doi.org/10.1186/1744-9081-2-33

9. Martinussen R, Hayden J, Hogg-Johnson S, Tannock R. A meta-analysis of working memory impairments in children with attention-deficit/hyperactivity disorder. J Am Acad Child Adol Psychiatry 2005;44(4):377-384. https://doi. org/10.1097/01.chi.0000153228.72591.73 
10. Murphy KR, Barkley RA, Bush T. Executive functioning and olfactory identification in young adults with attention deficit-hyperactivity disorder. Neuropsychology 2001;15(2):211-220. https://doi.org/10.1037/0894-4105.15.2.211

11. Visser J. Developmental coordination disorder: A review of research on subtypes and comorbidities. Hum Mov Sci 2003;22(4-5):479-493. https://doi.org/10.1016/j. humov.2003.09.005

12. Kaiser M-L, Schoemaker MM, Albaret J-M, Geuze RH. What is the evidence of impaired motor skills and motor control among children with attention deficit hyperactivity disorder (ADHD)? Systematic review of the literature. Res Dev Disabil 2015;36:338-357. https://doi.org/10.1016/j.ridd.2014.09.023

13. Zwicker JG, Missiuna C, Harris SR, Boyd LA. Developmental coordination disorder: A review and update. Eur J Paed Neur 2012;16(6):573-581. https://doi. org/10.1016/j.ejpn.2012.05.005

14. Ghanizadeh A. Predictors of postural stability in children with ADHD. J Attention Dis 2010;15(7):604-610. https://doi.org/10.1177/1087054710370936

15. Watemberg N, Waiserberg N, Zuk L, Lerman-Sagie T. Developmental coordination disorder in children with attention-deficit-hyperactivity disorder and physical therapy intervention. Dev Med Child Neur 2007;49(12):920-925. https://doi. org/10.1111/j.1469-8749.2007.00920.x

16. Fliers E, Rommelse N, Vermeulen SHHM, et al. Motor coordination problems in children and adolescents with ADHD rated by parents and teachers: Effects of age and gender. J Neural Trans 2007;115(2):211-220. https://doi.org/10.1007/s00702007-0827-0

17. Pienaar AE, Du Toit Y. Efficacy of different treatment methods for ADHD and associated neuromotor coordination difficulties. Afr J Phys Act Health Sci 2017; (Supplement 1.1):75-92

18. Pila-Nemutandani GR, Pillay BJ, Meyer A. Gross motor skills in children with attention deficit hyperactivity disorder. S Afr J Occ Ther 2018;48(3):19-23. https://doi.org/10.17159/2310-3833/2017/vol48n3a4
19. Kasparek T, Theiner P, Filova A. Neurobiology of ADHD from childhood to adulthood. J Atten Disord 2013;19(11):931-943. https://doi. org/10.1177/1087054713505322

20. Curatolo P, D’Agati E, Moavero R. The neurobiological basis of ADHD. Ital J Pediatric 2010;36(1):79. https://doi.org/10.1186/1824-7288-36-79

21. Conners CK. Conners' Rating Scales - Revised: Short Form. North Tonawanda, NY: Multi-Health Systems; 1997.

22. Wilson BN, Crawford SG, Green D, Roberts G, Aylott A, Kaplan BJ Psychometric properties of the Revised Developmental Coordination Disorder Questionnaire. Phys Occup Ther Pediatr 2009;29(2):182-202. https://doi. org $/ 10.1080 / 01942630902784761$

23. Henderson SE, Sugden D, Barnett AL. Movement Assessment Battery for Children-2. APA Psych Tests. Washington, DC: APA; 2007. https://doi. org/10.1037/t55281-000

24. Pearsall-Jones JG, Piek JP, Levy F. Developmental coordination disorder and cerebral palsy: Categories or a continuum? Humn Move Sci 2010;29(5):787-798. https://doi.org/10.1016/j.humov.2010.04.006

25. Bart O, Podoly T, Bar-Haim Y. A preliminary study on the effect of methylphenidate on motor performance in children with comorbid DCD and ADHD. Res Dev Disabil 2010;31(6):1443-1447. https://doi.org/10.1016/j. ridd.2010.06.014

26. Stray L, Stray T, Iversen S, Ruud A, Ellertsen B, Tønnessen F. The motor function neurological assessment (MFNU) as an indicator of motor function problems in boys with ADHD. Behav Brain Funct 2009;5(1):22. https://doi.org/10.1186/17449081-5-22

Accepted 7 May 2020. 